\title{
A comparison of two different techniques for deriving the quiet day curve from SARINET riometer data
}

\author{
J. Moro ${ }^{1}$, C. M. Denardini ${ }^{1}$, E. Correia ${ }^{1,2}$, M. A. Abdu ${ }^{1}$, N. J. Schuch ${ }^{3}$, and K. Makita \\ ${ }^{1}$ Instituto Nacional de Pesquisas Espaciais - INPE, São José dos Campos - SP, Brazil \\ ${ }^{2}$ CRAAM - Mackenzie University, São Paulo - SP, Brazil \\ ${ }^{3}$ Southern Regional Space Research Center, Santa Maria - RS, Brazil \\ ${ }^{4}$ Takushoku University, Japan
}

Correspondence to: J. Moro (juliano@dae.inpe.br)

Received: 13 July 2011 - Revised: 4 April 2012 - Accepted: 18 July 2012 - Published: 14 August 2012

\begin{abstract}
In this work, an upgrade of the technique for estimating the Quiet Day Curve (QDC) as proposed by Tanaka et al. (2007) is suggested. To validate our approach, the QDC is estimated from data acquired by the Imaging Riometer for Ionospheric Studies (IRIS) installed at the Southern Space Observatory (SSO/CRS/CCR/INPE - MCT, $29^{\circ} 4^{\prime} \mathrm{S}$, $53^{\circ} 8^{\prime} \mathrm{W}, 480$ m a.s.l.), São Martinho da Serra - Brazil. The evaluation was performed by comparing the difference between the QDCs derived using our upgrade technique with the one proposed by Tanaka et al. (2007). The results are discussed in terms of the seasonal variability and the level of magnetic disturbance. Also, the cosmic noise absorption (CNA) images for IRIS data operated at SSO was built using both the techniques aiming to check the implications of the changes in the methods of QDC determination on the CNA that resulted from it.
\end{abstract}

Keywords. Ionosphere (Instruments and techniques)

\section{Introduction}

Ionospheric absorptions have been studied for many years using a variety of experimental techniques. One technique for absorption measurements involves the continuous recording of cosmic radio noise that has passed through the ionosphere. This is the purpose of the Relative Ionospheric Opacity Meter (riometer), which uses a stable receiver on the ground operating at a frequency usually between 20 and $60 \mathrm{MHz}$.

The riometer has been used to study the cosmic noise absorption (CNA) since the early 1960s (Little and Leinbach, 1959; Lusignan, 1960; Abdu et al., 1967, 1973; Nishino et al., 1993, 2002, 2006). The CNA generally occurs in the Dregion of the ionosphere, which is the region with the highest concentration of molecular gases. Any free electron present in this region will have its motion affected by the energy absorbed from a propagating cosmic noise. Due to the high collision rates at these altitudes, this extra motion will lead to an extra energy transferred to the heavier particle, thereby causing the CNA (Browne et al., 1995).

The CNA is measured relative to the amplitude of cosmic noise signal received under quiet ionospheric conditions, which makes it important to obtain a consistent Quiet Day Curve (QDC). The methodology of determining the QDC has been discussed extensively by several authors (Mitra and Shain, 1953; Lusignan, 1960; Steiger and Warmick, 1961; Fredriksen and Dyce, 1967; Heisler and Hower, 1967; Armstrong et al., 1977; Krishnaswamy et al., 1985; Tanaka et al., 2007). Despite the many works on the subject since the 1960s, a reliable methodology to derive the QDC from riometer data is still a puzzling task. The cosmic noise detected by riometers presents strong day-to-day and seasonal variability, as well as variability due to the solar cycle activity and magnetic disturbances.

In the present paper, a new mathematical approach for determining the QDC from the South America Riometer Network (SARINET) data is proposed based on two criteria: riometer data selection accordingly to the geomagnetic activity and data cleaning with respect to the electromagnetic interferences. In order to check the results obtained, our technique is compared with that derived by Tanaka et al. (2007), which has been used to estimate the QDC from SARINET data. The 
differences in terms of the CNA images obtained from these techniques are presented and discussed.

\section{The Imaging Riometer for Ionospheric Studies (IRIS)}

The discussions with respect to the differences between our technique and that proposed by Tanaka et al. (2007) are based on the analysis of the data acquired by the IRIS (Detrick and Rosenberg, 1990) installed at SSO, which form part of SARINET. Seven IRIS and 17 single beam riometers from SARINET are currently operational in the American sector (Southern Hemisphere) and in Japan (Northern Hemisphere). Accordingly, coordinated IRIS observations by the SARINET in the Southern and Northern Hemispheres would contribute to the study of ionosphere behaviour due to the energetic particles precipitation in the South America Magnetic Anomaly (Abdu et al., 2005) in low and middle latitudes.

The IRIS antenna system of SARINET consists of a twodimensional co-linear co-array of 16 half-wavelength dipoles at the operating frequency of $38.2 \mathrm{MHz}$. Since the array is a coco, the separation between the antennas is a halfwavelength (Balsley and Ecklund , 1972). One of the twodimensional array is aligned in the geographic north-south $(\mathrm{N}-\mathrm{S})$, while the others is in quadrature and, therefore, is set in the east-west $(\mathrm{E}-\mathrm{W})$ direction. The field-of-view of the IRIS at $100 \mathrm{~km}$ of altitude is approximately $330 \mathrm{~km}$ in both $\mathrm{N}-\mathrm{S}$ and $\mathrm{E}-\mathrm{W}$ directions (Nishino et al., 2002). By connection of the dipole array with a Butler-matrix phasing circuit and four channel radio receivers, $4 \times 4$ two-dimensional beams with a half-power beam-width of $22^{\circ}$ are directed upward to the ionospheric area centred at the zenith. The basic operation of the IRIS consists of acquiring levels of voltage from each of the 16 beams in the four receivers. Each four beams are read at one time, meaning that four acquisitions are necessary to get all the 16 levels of voltage from the 16 beams. These voltages are then converted in a binary coded and sequentially stored in a binary file. Each file contains one day of the riometer data within 17 sets of information: one for the time line and one for each of the 16 beams.

\section{Techniques for estimate QDC}

Since the cosmic noise level acquired by riometers continuously fluctuates due the ionosphere density variations, it is not possible to achieve an ideal situation of zero absorption. Therefore, statistical techniques are employed to estimate the QDC. In the early studies, the techniques were to scale the daily charts for a sufficient period of time, transfer these values to the proper sidereal time (ST) and compare all values for a giving sidereal hour. The highest reliable values for each hour become point on the QDC (Mitra and Shain, 1953; Lusignan, 1960; Steiger and Warmick, 1961; Fredriksen and Dyce, 1967). These methodologies as- sume that the highest values correspond to a condition of zero absorption. However, even on undisturbed periods the minimum absorption does not go to zero and has seasonal variations. Accordingly, some studies more refined to estimate QDC have been available. The technique proposed by Heisler and Hower (1967) consists of an estimate of the QDC using the early morning riometer data for a giving year. Only riometer data acquired from 00:00 until 06:00 (local time) is considered, since the ionospheric absorption is minimum at such times. However, the level of the curve must be adjusted to correspond to the condition of minimum absorption and this technique requires an extensive riometer database. Armstrong et al. (1977) proposed that the better definition of QDC would be the values corresponding to the inflection point on the high-signal side of the peak of the data distribution for that interval. This method was implemented by $\mathrm{Kr}$ ishnaswamy et al. (1985) to estimate the QDC by computer, which avoid much of the subjectivity involved in the previews studies. Finally, the technique proposed by Tanaka et al. (2007) estimate the QDC values in a certain time-period using a percentual criteria from the cosmic noise intensity distribution, applied to SARINET data. Moreover the QDC curves are determined by the running average of the QDC values. Nevertheless, Tanaka's technique is not appropriated during times of persistent solar radio emissions or man-made interferences. In the next section, we will discuss Tanaka's technique and the modifications suggested for us in order to estimate the QDC from SARINET data.

\subsection{Tanaka's technique for estimate QDC}

The basic data processing starts with the data stored in daily binary files being converted to power, for each month of IRIS operation. No distinction is made regarding the level of magnetic disturbance so far. Due to the Earth's translation, the antenna beam takes less than one Earth's rotation to cover the same area of the sky that it explored during the previous solar day. Indeed, it takes $23 \mathrm{~h} 56 \mathrm{~min} 4 \mathrm{~s}$, which constituted a sidereal day (Duffett-Smith, 1979). Therefore, all the analysis of the riometer data is based on ST, instead of the universal time (UT), and the data acquired in UT is converted to ST. Data from all days of the month are grouped by beam following the ST line. Thereafter, all levels of voltage for each beam corresponding to the same sidereal minute are averaged. This leads to the sidereal time variation of the monthly averaged level of voltage for each beam $(\mu)$. Similar procedure is applied to the standard deviation $(\sigma)$ as well.

For each beam, the sidereal time variation of the monthly averaged level of voltage and the corresponding standard deviation are used to select the valid voltage levels measured by the IRIS at each sidereal minute. The valid data are constrained to the range of $\mu \pm 3 \sigma$, and all data points out of range are discarded. A new $\mu$ and $\sigma$ are then calculated with the valid data and a new selection of data in the range $\mu \pm 3 \sigma$ is performed. This procedure repeats until no data to 

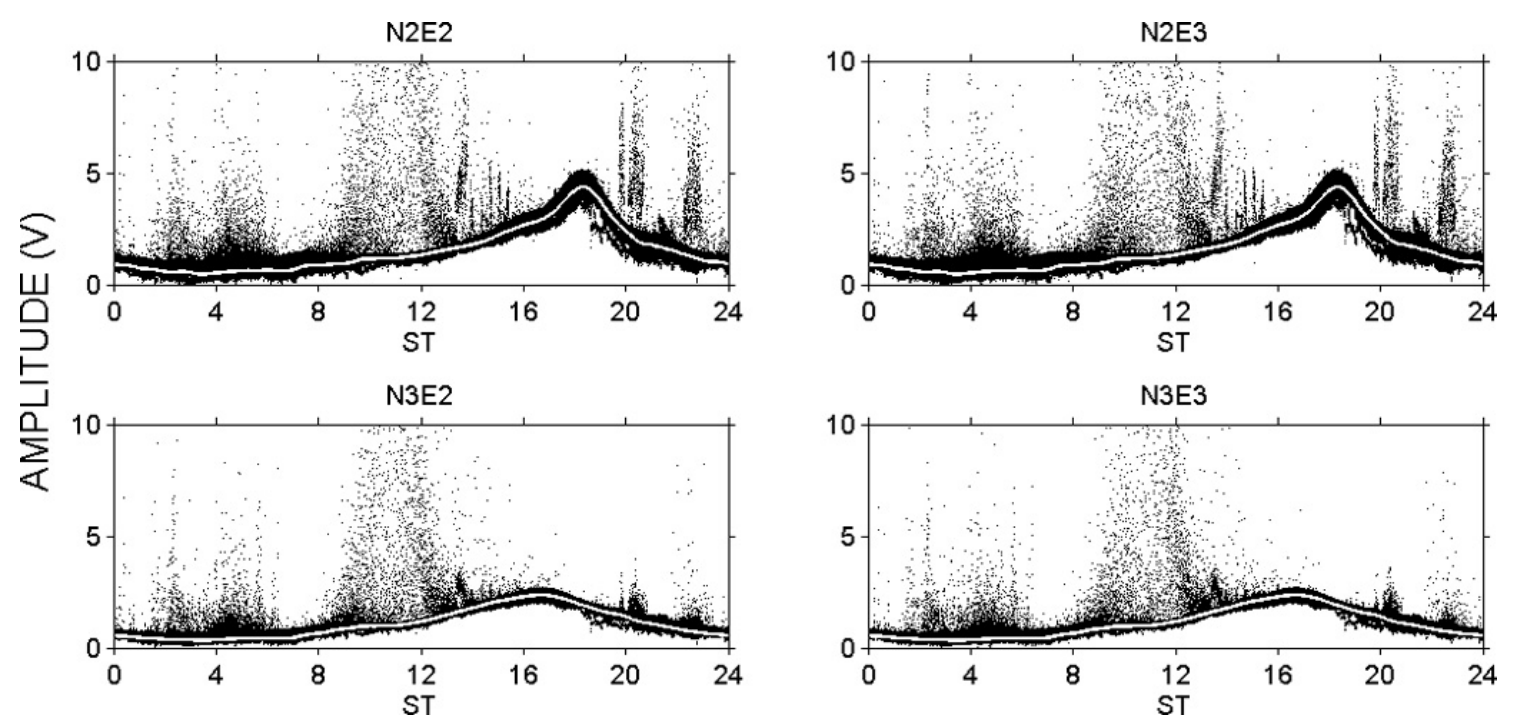

Fig. 1. Example of the diurnal variation of cosmic noise acquired by IRIS installed at SSO - Brazil for March 2010 (black dots) using the 4 most central beams superimposed by (white line) the QDC derived for this period based on the Tanaka's technique (N: North, E: East).

be discharged. Using the final $\mu$ and $\sigma$, Tanaka et al. (2007) take $80 \%$ of the $\mu$ added by $3 \sigma$ as the pre-QDC value at each minute. Finally, the QDC for each beam is defined as the 30 points running average of this last result.

Figure 1 shows in white the four QDCs obtained for the four most central beams, determined for March 2010, as an example of the Tanaka's technique. The vertical axes of each graph are set in voltage levels and the sidereal time runs along the horizontal axes. The letters and number over each graph identify the antenna for which the QDC is determined.

After the QDC estimation we calculated the CNA during a period of interest. The CNA (in $\mathrm{dB}$ units) is calculated using the ratio of the noise power during quiet conditions $(\mathrm{P})$ to the noise power during a period of interest $\left(P^{\prime}\right)$, as per Eq. (1):

$\mathrm{CNA}(\mathrm{dB})=10 \log \left[P / P^{\prime}\right]$.

From the individual CNA for each beam, we created a spatial image of the CNA for a given time. The two-dimensional $4 \times 4$ data are converted to two-dimensional $16 \times 16$ data, in order to obtain smooth coloured images of the absorption representing the 16 elements of the beam. Therefore, the $330 \times 330 \mathrm{~km}(\mathrm{~N}-\mathrm{S}$ and $\mathrm{E}-\mathrm{W}$ directions $)$ image has an artificial spatial resolution of about $21 \times 21 \mathrm{~km}$ at a reference height of $100 \mathrm{~km}$ in the ionosphere overhead the IRIS.

Finally, it is important to mention that despite having the images calculated with the QDC from the 16 beam, we based our comparative analyses of the differences between the methods of estimating the QDC on the results from the four most central beams only. For this analyses we consider the antennas directed close to the zenith (N2E2, N2E3, N3E2 and N3E3). This approach constrains the data collected to the most vertical beams of the IRIS only, which intended to avoid the local electromagnetic interference as much as possible.

\subsection{Description of the new approach suggested for the QDC estimate}

There are two basic differences between the Tanaka's technique to define the QDC and the new approach we are proposing. The first difference is in the selection of IRIS data according to the geomagnetic activity. Accordingly, in our approach the Kp index (Rostoker, 1972; Wrenn et al., 1987) is used for selecting quiet time data only $(\mathrm{Kp} \leq 3)$. Also, the IRIS data collected on a day before and after a geomagnetic disturbance period is eliminated to avoid possible contamination from magnetic disturbances. The second difference lies on the qualification of the electromagnetic radio interference on the cosmic noise measurements. The electromagnetic interferences may occur due to the presence of man-made interferences, thunderstorms or solar noise bursts. These bursts can affect the estimation of the QDC leading to an increase of the QDC level around the sidereal minute when the burst occurred.

The electromagnetic interferences are characterised by large amplitudes when compared with the default signal obtained by IRIS and often exceed $10 \mathrm{~V}$ (the maximum level of acquisition of the analog-to-digital converter). In order to eliminate them, we propose to analyse all levels of voltage for each beam inside each 10 sidereal minute interval before running the analysis of $\mu \pm 3 \sigma$. For every interval along the day, we group all the measurements of voltage levels made for all the selected quiet days and take the median $(\alpha)$. Thereafter, every single measurement of voltage level is compared with $\alpha$. If the absolute difference surpasses $\pm 0.15 \mathrm{~V}$ (empirical value established during ours analyses) this point is labelled as "not valid" and is not consider in the $\mu \pm 3 \sigma$ analyses. However, our approach fails to 

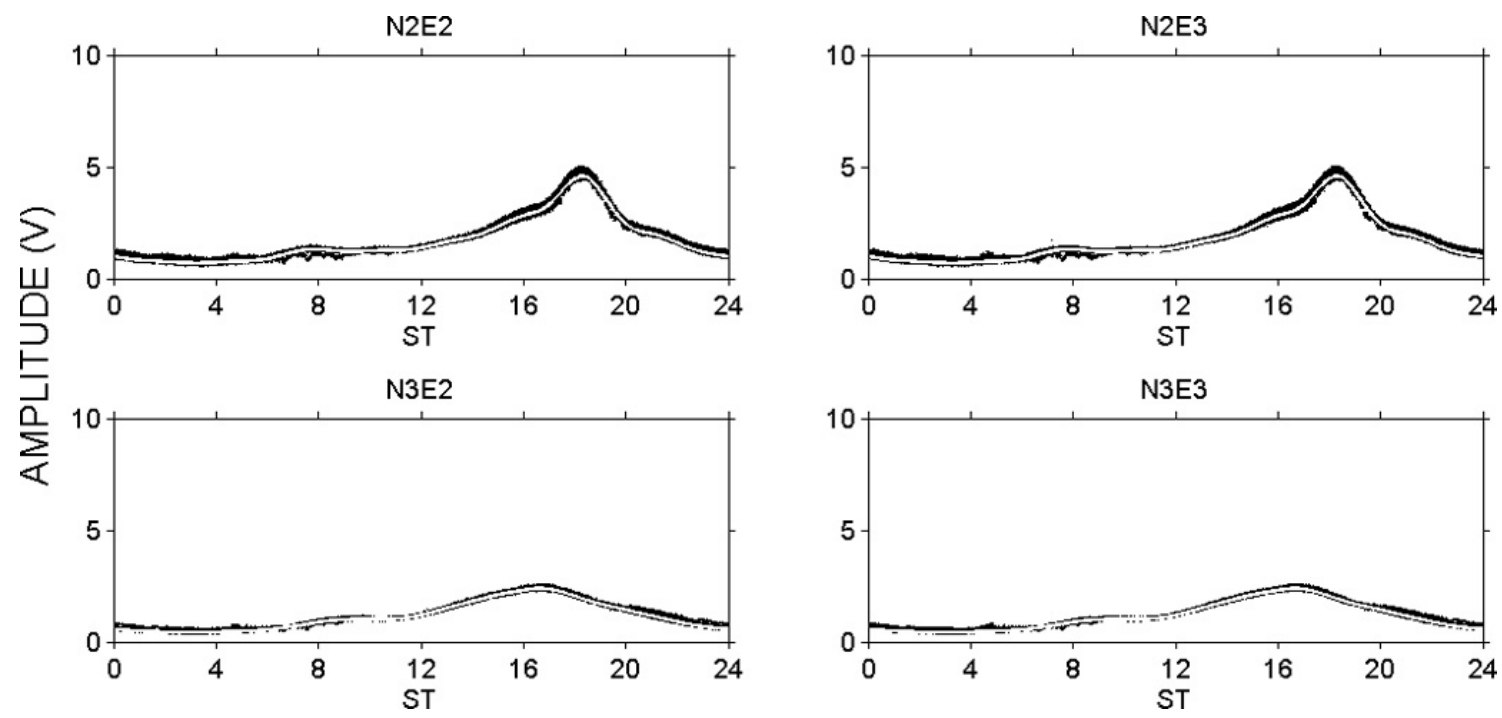

Fig. 2. Same as Fig. 1, but considering the criteria that we adopted (black dots) superimposed by (white line) the QDC derived for this period.

eliminate the electromagnetic interference when it lasts more than 10 min. Accordingly, Fig. 2 shows the results of four QDCs (1 per graph) for the four most central beams determined from the same data as Fig. 1, but using our technique in this case, which takes into account the two above mentioned criteria. The vertical and horizontal axes of each graph are the same as those shown in Fig. 1, as are also the letters and number over each graph.

\section{Results and discussions}

Figure 1 provides an example of the diurnal variation of cosmic noise intensity acquired during all the days of March 2010 (black dots) by IRIS, superimposed by the QDC (white line) derived in the same period based on the Tanaka's technique. It intends to illustrate the typical information obtained about the cosmic noise received by the riometers and the presence of electromagnetic interferences causing the dispersion of the signal along the day that are mainly around 04:00, 12:00, 20:00 and 22:00 ST. The graphs in Fig. 2 show the same dataset as that of Fig. 1, but filtered and cleared taking into account the two criteria that we adopted: selection of data acquired on quiet days only and with no electromagnetic interferences. The new QDCs derived from our approach are also superimposed on the graphs. It should be noticed that the data dispersion after our selection criteria is drastically reduced.

Following these two examples, we have calculated the QDC using the approach proposed by Tanaka et al. (2007) for all the dataset acquired by the IRIS installed at SSO, from January 2007 to January 2011, totaling 44 months of data (around the last solar minimum), meaning 44 monthly QDCs. We also estimate the 44 QDCs using our approach from the same dataset. Afterwards, we have taken the dif- ference between the individual corresponding QDCs derived by both methods. The evolution of the mean monthly difference is presented in the continuous thich line of the graph of Fig. 3. Additionally, we have calculated the QDCs using only one of the two criteria in our approach to check the impact of each one in the QDC determination. The differences were also taken and the monthly mean difference is also placed in the Fig. 3: the continuous thin line shows the evolution of the mean monthly difference between the method proposed by Tanaka et al. (2007) and that obtained when the electromagnetic interference elimination is implemented; and the dotted line shows the evolution of the monthly difference between the method proposed by Tanaka et al. (2007) and that obtained using the data selection based on magnetic disturbance criterion only. The reader should be aware that each point in the graph is calculated by the percentual difference between the levels of noise power of the QDC obtained by Tanaka's technique and that of the QDC obtained regarding the specific criteria being used. For example, the value obtained for the mean differences between the two techniques for March 2010 in Fig. 3 is calculation by the percentual difference between the QDCs in Figs. 1 and 2. The same was repeated for all the other months of the analysis.

In order to discuss the results shown in the graph of Fig. 3, we have considered two hypotheses about the current method to estimate the QDC. The first hypothesis is that the method proposed by Tanaka et al. (2007) fails. Following this assumption any improvement in the data selection or data clearing should improve the result. Therefore, the new QDC should differ from the original one, leading the line in Fig. 3 to separate from the $0 \%$ level. Second hypothesis is based on the consideration that the method proposed by Tanaka et al. (2007) is a valid and successful one. In this case, the closer 


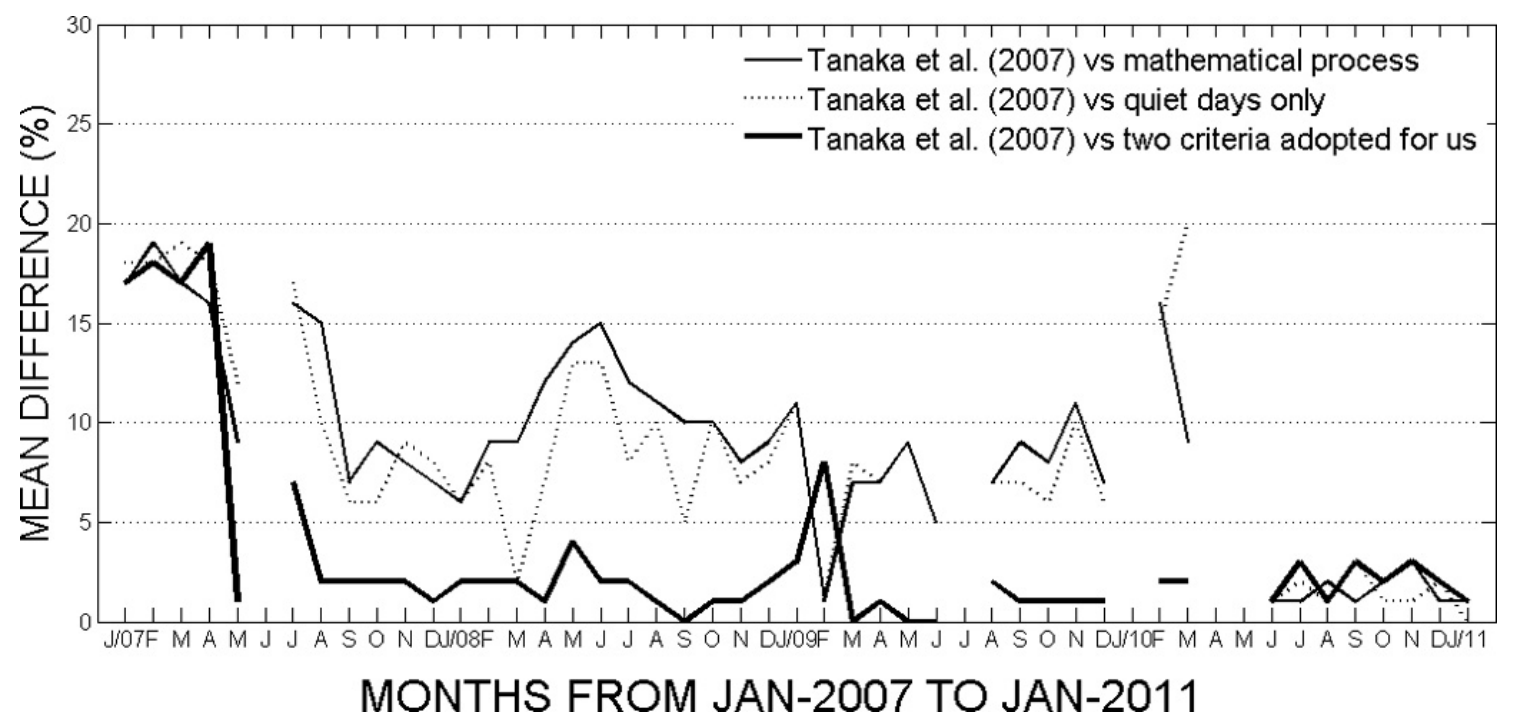

Fig. 3. Seasonal variability of the mean difference between the QDCs derived with the approach proposed by Tanaka et al. (2007) and that obtained from the same approach but with different criteria: eliminating electromagnetic interference (continuous thin line); considering quiet days only (dotted line); and eliminating electromagnetic interference and selecting data from quiet days only (continuous thick line).

the line in the graph of Fig. 3 of the $0 \%$ level is, the better the possible result.

Considering the first hypotheses, the data selection based on a mathematical process (continuous thin line) improved the determination of QDC, since we had a difference of about $10 \%$ in average (from graph of Fig. 3). Also a simple data selection based in data acquired during quiet days was able to improve the estimation of the QDC as well, since this new QDC differs from the QDC derived by the method of Tanaka et al. (2007) by $8 \%$ in average.

Still considering the first hypotheses, once we are using very explicit physical criteria for data selection in our methods, i.e., qualification of the electromagnetic radio interference on the cosmic noise measurements and the classification of data according to the geomagnetic activity, a contribution of both should logically lead to an even bigger separation of the QDC derived in this way from that one obtained by the use of Tanaka's method. In other words, the difference observed in the graph should increase. However, the combination of our two data selection criteria brought this difference down, as can be seen by the continuous thick line in Fig. 3 .

Therefore, the first hypothesis seems not to be the valid one and the second assumption, i.e., that the method proposed by Tanaka et al. (2007) is valid and successful method gains support. But it does not mean that it is better than our two steps together. It does mean that the statistical method proposed by Tanaka et al. (2007) is better than just a single mathematical process criterion or selection of IRIS data according to the geomagnetic activity. Indeed, our results show that the current technique to calculate the QDC seems to include the reduction of electromagnetic interferences discussed in Sect. 3.2 and the reduction of the implications of the presence of data acquired in disturbed days $(\mathrm{Kp}>3)$, even if it is not explicit. So, the best "locus" of the solutions of determining the QDC should fall between the continuous thick line and the $0 \%$ level of Fig. 3.

However, we consider our method more accurate since it exposes the physical considerations behind the technique. The difference between Tanaka's technique to define the QDC and the new approach proposed here becomes more important in period when the dataset is more problematic like that data acquired during disturbed time. Take, for instance, the period from January to April 2007 and in February 2009 when the cases were more problematic. At this point it is important to recall that the graph of Fig. 3 shows differences and that we have chosen the Tanaka's technique as a frame of reference $(0 \%$ level), which does not mean it is fail proof. So, the differences obtained during the period between January to April 2007 and in February 2009 only mean that the two techniques strongly differ from each other. Therefore, we believed that the magnetic condition leaded to a separation from $0 \%$ level in the graph of Fig. 3 not because our method failed, but due to a bad determination of the QDC by the method of Tanaka et al. (2007) related to severe magnetic disturbances all over the period. In order to check this last statement, we performed an analysis of the differences between the method proposed in the present study and that one used by Tanaka et al. (2007) along a sidereal day. Additionally, we analysed the impact of the method proposed by Tanaka et al. (2007) in the CNA images comparing with the images built with the QDCs proposed in the present work. 

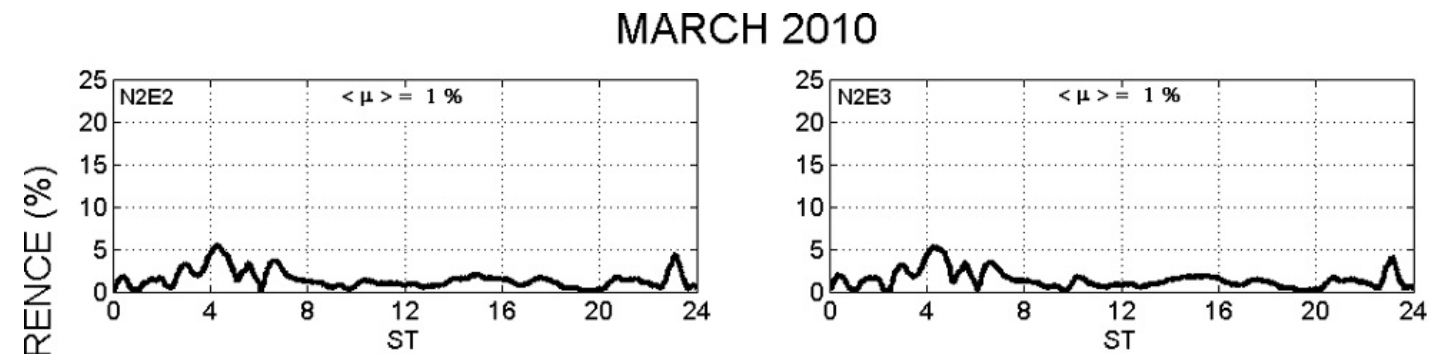

(a)
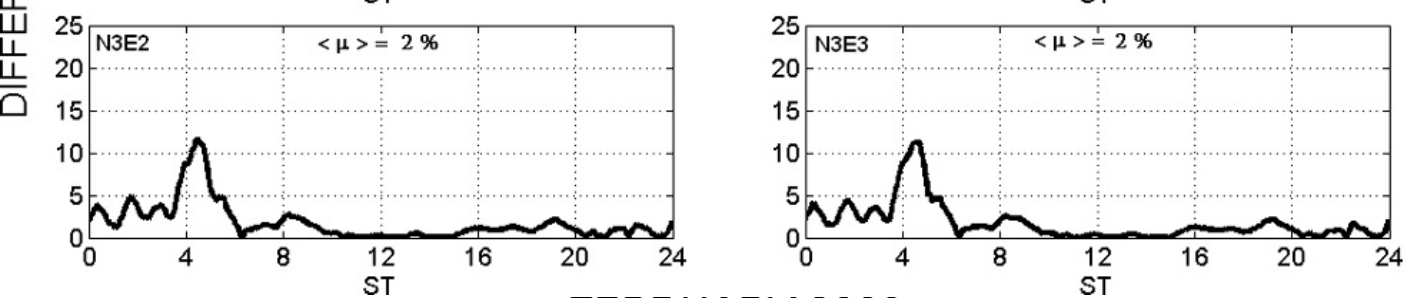

FEBRUARY 2009
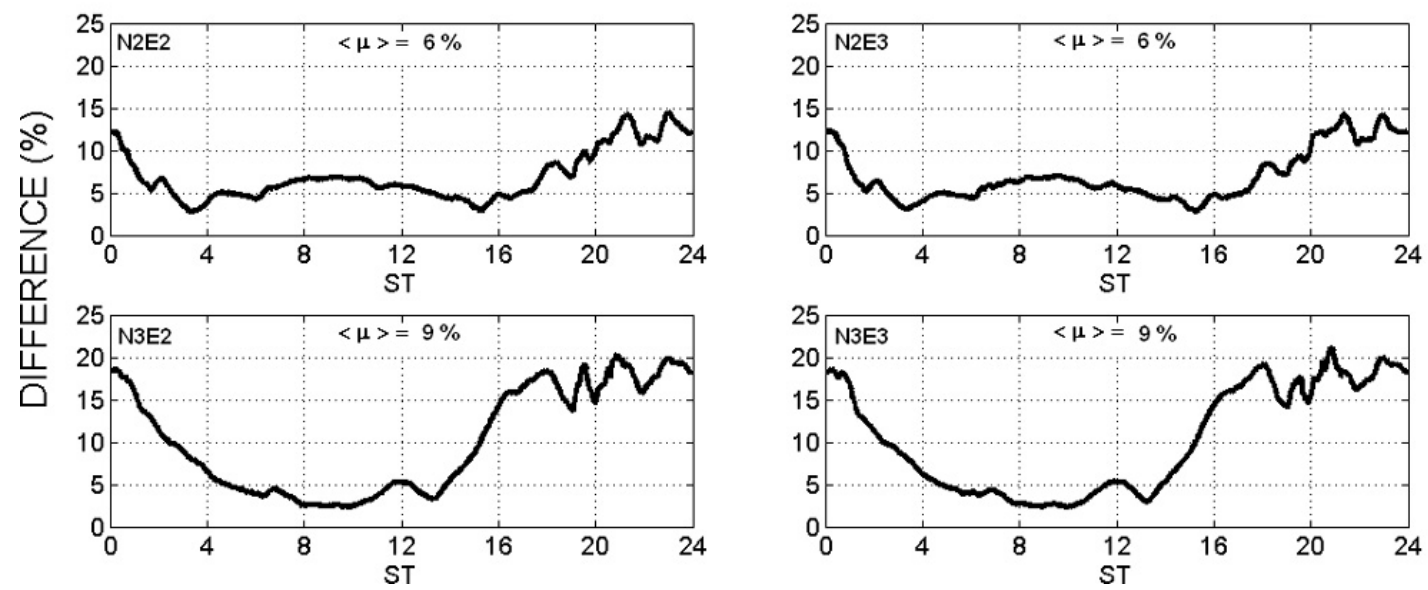

(b)

Fig. 4. Diurnal variation of the percentual difference between the two techniques for a sidereal day, calculated for all data collected by four beams direct to the zenith of the IRIS installed at SSO (a) in March 2010 and (b) February 2009. The average throughout the day, which gives the monthly averaged value in Fig. 3, is also presented.

\subsection{Analysis of the differences for a sidereal day}

Some implications of using an explicit physical meaning for selection of the IRIS data are more clearly visible when we analyse the difference between the two techniques for a sidereal day. In order to exemplify that, we investigate two opposite cases, March 2010 and February 2009, when the differences between monthly QDCs are smaller and bigger, respectively. The QDCs from March 2010 was chosen to match with the examples presented in Figs. 1 and 2, while the QDCs from February 2009 represent a peak in the graph of Fig. 3. The results are shown in the graphs of Fig. 4. It presents the percentual difference between the QDCs calculates with our approach and that one using the Tanaka's technique in the vertical axes. The sidereal time is placed in the horizontal axes. We also calculated the average throughout the day, which is placed in the upper portion of each graph. It should be remembered that the average of these values gives the monthly averaged value as presented in Fig. 3. The let- ters and number on left top corner of each graph identify the antenna that was used to collect the data.

The case of March 2010, when the QDCs differ by < $\mu>=2 \%$, have a diurnal variation with values much greater than that, indeed. We can see in Fig. 4a that around 04:00 ST the difference between the QDCs is lower than $6 \%$ in the antennas N2E2 and N2E3, but more than $10 \%$ in the N3E2 and N3E3. This big difference comes from strong electromagnetic interference that is clearly seen in the graphs of Fig. 1 close to 04:00 ST, which affected the QDC obtained by Tanaka's technique, and were removed with our new approach (Fig. 2).

For February 2009, when the QDCs differ by $<\mu>=$ $9 \%$, the monthly averaged diurnal variation reaches values higher than that obtained in March 2010. In the first four hours the graphs for the antennas N3E2 and N3E3 show differences as high as $20 \%$, while the other graphs present differences of $\approx 15 \%$. Also, there is a clear reduction of the difference between 00:00 and 04:00 ST, down to level of 5\%. 

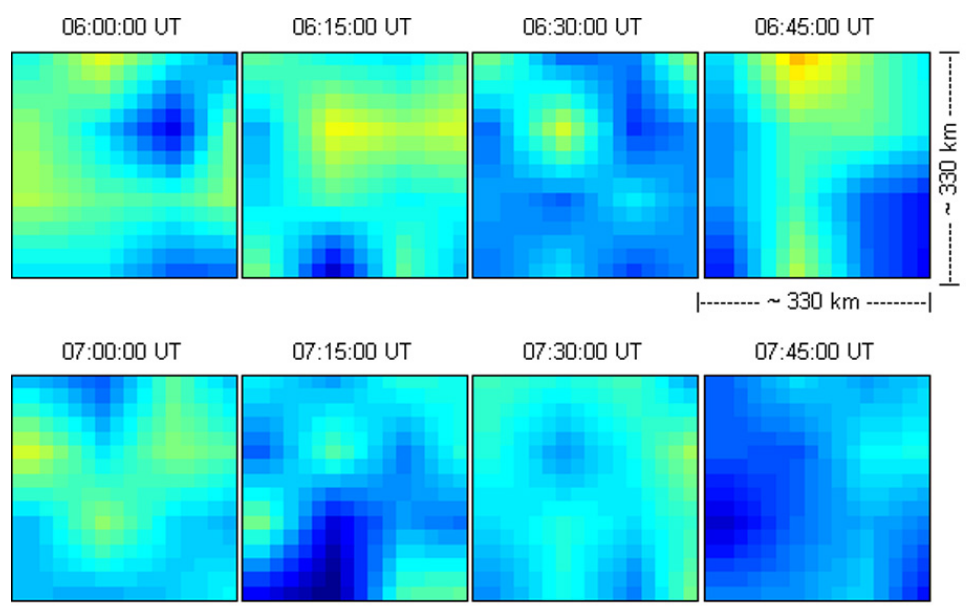

(a)

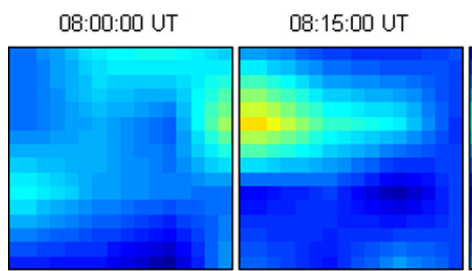

08:30:00 UT

08:45:00 UT
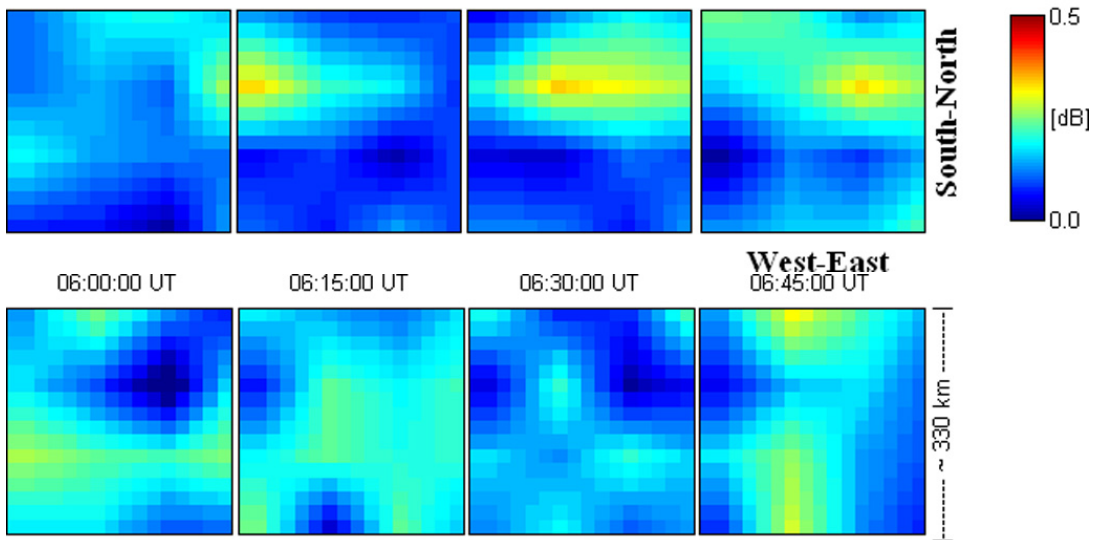

06:15:00 UT

06:30:00 UT

West-East
06:45:00 UT

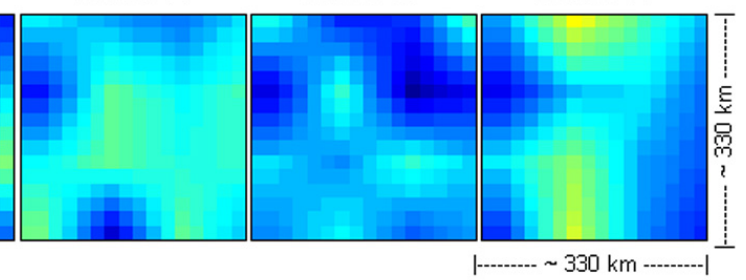

07:00:00 UT

07:15:00 UT

07:30:00 UT

07:45:00 UT

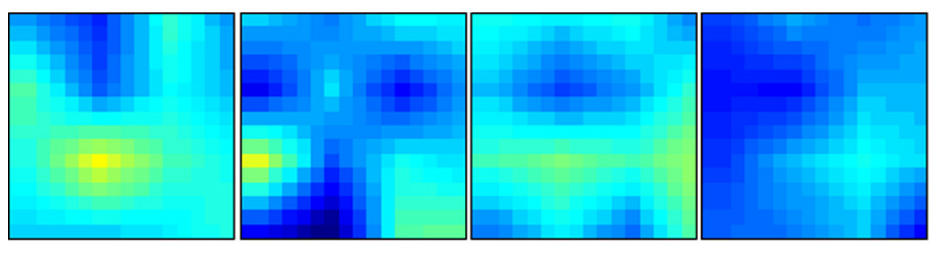

(b)

08:00:00 UT

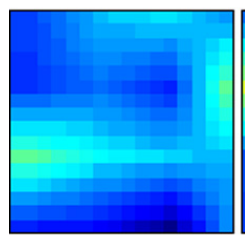

08:15:00 UT

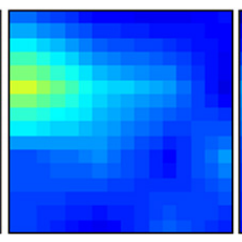

08:30:00 UT

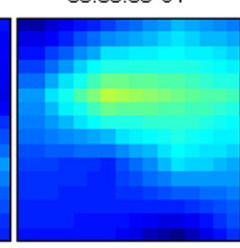

08:45:00 UT

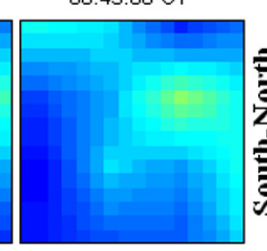

West-East

Fig. 5. Images of CNA calculated every $15 \mathrm{~min}$ at SSO - Brazil on 28/Mar./2010, using (a) our technique to derive the QDC and (b) the QDC obtained with the Tanaka's technique.

After that, the differences stay around 5\% until 16:00 ST. After 16:00 ST the difference of the QDC derived by our method to the QDC based on Tanaka's method start to increase again up to 20:00 ST. In all the graphs the differences come back to its midnight values after that time. These strong differences present during 16:00-24:00 ST along a sidereal day on February 2009 are caused in most part by the local electromagnetic interferences like the example presented in 

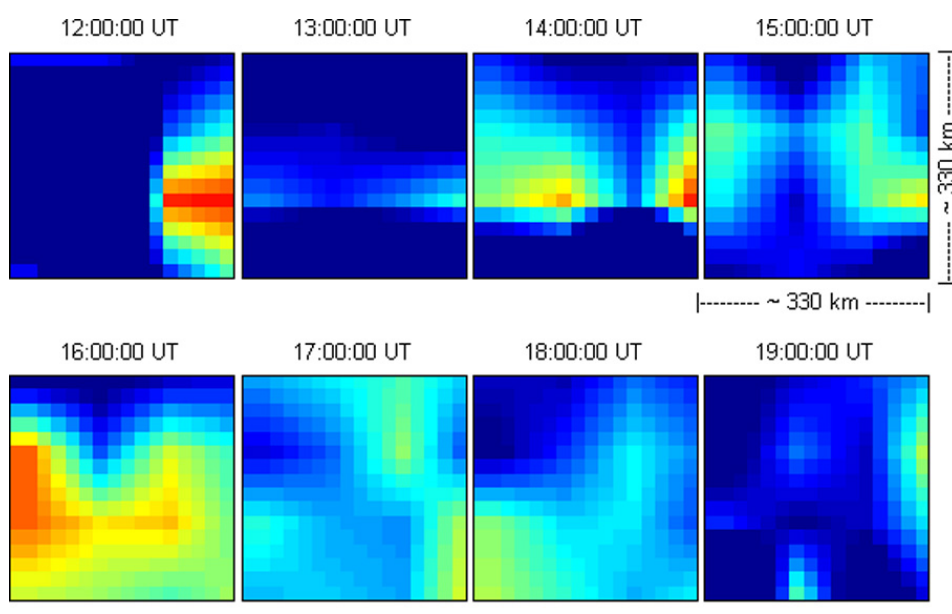

(a)

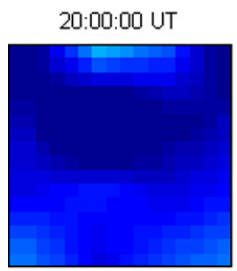

21:00:00 UT

22:00:00 UT
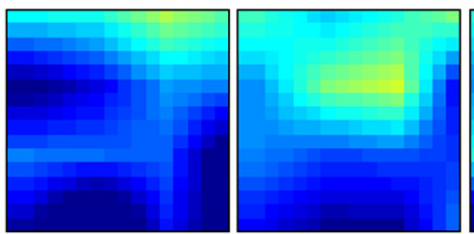

23:00:00 UT
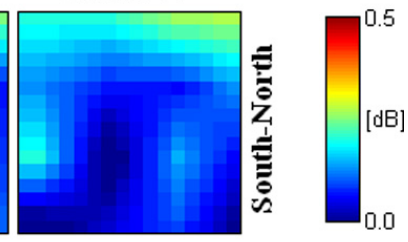

12:00:00 UT

13:00:00 UT

14:00:00 UT

West-East
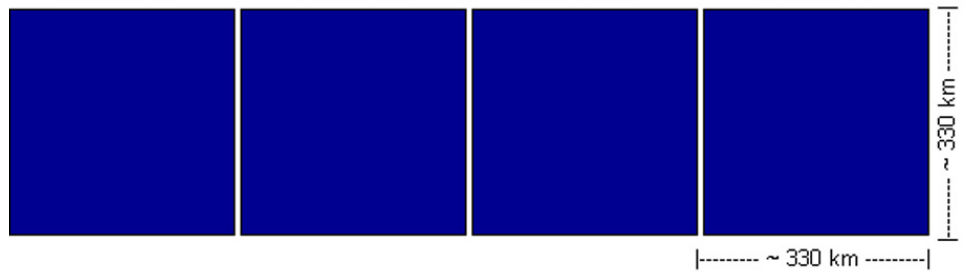

16:00:00 UT

17:00:00 UT

18:00:00 UT

19:00:00 UT
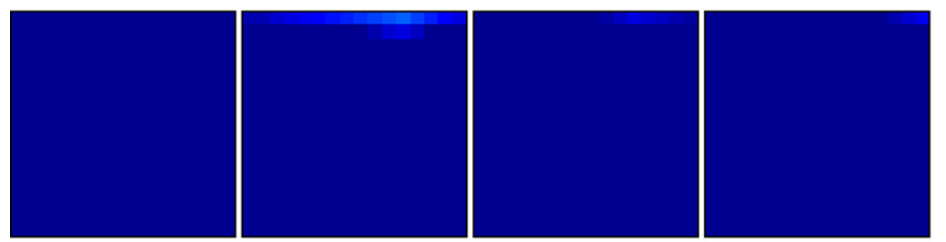

(b)

20:00:00 UT

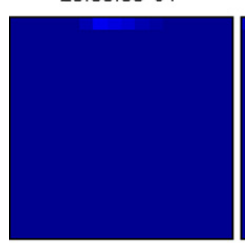

21:00:00 UT

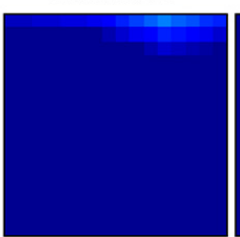

22:00:00 UT

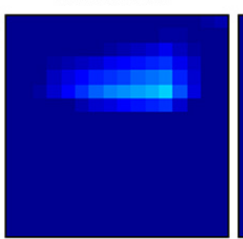

23:00:00 UT

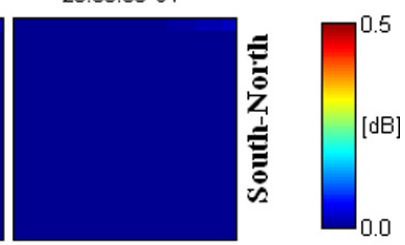

West-East

Fig. 6. Images of CNA calculated every $1 \mathrm{~h}$ at SSO - Brazil on 4 February 2009, using (a) our technique to derive the QDC and (b) the QDC obtained with the Tanaka's technique.

the Fig. 1 (but not shown here) present in the cosmic noise data and reinforce the conviction of the good performance of our technique. Finally, the monthly averaged diurnal variation discussed with the examples presented above, which af- fects the QDCs by more than $10 \%$ in March 2010 and around $20 \%$ in February 2009, results in not confident CNA images. The influence of not confident QDCs in the CNA Analysis will be discussed in the next section. 


\subsection{Implication of our approach in determine the CNA images}

In order to check the implications of each technique for deriving the QDC in the determination of the CNA, we built 2-D-CNA images for the same period as discussed in Fig. 4, i.e., March 2010 and February 2009. The CNA images calculate with QDC derived for our approach is presented in Figs. 5a and 6a (named "a" sequences), while the CNA images estimate with the Tanaka's technique are present in Figs. $5 \mathrm{~b}$ and $6 \mathrm{~b}$ (named "b" sequences). Each image represents $\approx 330 \times 330 \mathrm{~km}$ in both $\mathrm{N}-\mathrm{S}$ and $\mathrm{E}-\mathrm{W}$ directions (with North at the top and East on right-hand side of each image), with spatial resolution of about $21 \times 21 \mathrm{~km}$ at $100 \mathrm{~km}$ altitude (see Sect. 3.1). The absorption intensity of the images is represented by the colour-coded bar $(0$ to $0.5 \mathrm{~dB})$ on the right-hand side of the last images. The CNA images show clear weak events (less than $0.5 \mathrm{~dB}$ ) of ionospheric absorption. However, the physical meaning of them will not be addressed in the present paper. The images are included here only to show the implications of the technique used to obtain the QDC in the CNA images.

The series of absorption images obtained every $15 \mathrm{~min}$ from 06:00 to 08:45 UT on 28 March 2010 are shown in the Fig. 5. A first look at these images reveals some irregularly distributed absorption regions along the analysed period in both sequences "a" and "b". In addition, the absorption regions seen in the "a" sequence can also be identified in the "b" sequence. However, a visual comparative analysis shows that all these regions seem to be more intensive in the "a" sequence. Taking the images at 06:15, 06:45 and 08:30 UT as examples, it is possible to infer that all these images have regions with enhanced intensity higher or equal to $0.3 \mathrm{~dB}$ in the "a" sequence. The same is not true for the " $b$ " sequence, where the intensity did not surpass $0.2 \mathrm{~dB}$ in the best case. Now, analysing the blurred case of the image at 06:00 UT, we see that our technique for determining QDC allows us to infer that there is a connection between the two absorption regions (the northern localised and the southern stretched band). The image from the "a" sequence shows a clear north-west connection while the image from the " $b$ " sequence shows no connection at all. Therefore, we can state that, even in a case of reasonable good agreement between the techniques for estimate the QDC, there still are some discrepancies in the CNA determination and it seems that our technique provides more accurate results.

The series of absorption images obtained every $60 \mathrm{~min}$ from 12:00 to 23:00 UT on 4 February 2009 are shown in the Fig. 6. The difference between the " $a$ " and " $b$ " sequences are dramatically evident in the present case. The "a" sequence shows a series of absorption regions along the analysed period (with some of them having intensity up to $0.5 \mathrm{~dB}$ ), while the "b" sequence barely shows at 22:00 UT a single localised absorption region. The most expressive event seen at 16:00 UT in the "a" sequence simply does not appear in the " $b$ " sequence. We attribute this to the strong electromagnetic interference in the data (not shown here), which were not identified by the Tanaka's statistical approach technique. Therefore, if reasonable agreement in the technique for estimate QDC lead to small discrepancies in the CNA, the few examples above show that there could be huge differences when the data were not properly treated for the QDC estimate. It reinforces our conviction that the technique based on physical meanings for data cleaning appears to produce a better QDC when compared with Tanaka's technique. The gain achieved from this is that greater detail in the structure of ionospheric absorption is revealed, as shown by Fig. 6 .

\section{Conclusions}

We propose an approach for deriving the QDC from SARINET riometer data, which improves the accuracy of CNA images. It is based on the classification of IRIS data according to the geomagnetic activity and the qualification of the electromagnetic radio interference. We have calculated the QDC using 44 months of data with this new approach and we compared with QDC estimated by the approach proposed by Tanaka et al. (2007). The differences between Tanaka's technique to define the QDC and the new approach proposed in this work becomes more important in period when the dataset is more problematic, e.g., data acquired during disturbed times. The percentage difference between the different techniques reach $20 \%$ in some cases. Some implications of using our criteria for selection for the IRIS data are clearly observed when we analyse the differences along a sidereal day. We also checked the implications of each technique for deriving the QDC in the determination of the 2-D-CNA images and we see that even in a case of reasonable good agreement between the techniques for estimate the QDC, there were still some discrepancies in the 2-D-CNA images determination. Also, when data were not properly treated for the QDC estimate the discrepancies could be huge. For all these reasons, we are convinced that our criteria for IRIS data selection and cleaning provided a significant improvement to the original technique proposed by Tanaka et al. (2007).

Acknowledgements. J. M. thanks CNPq/MCT for supporting his master programme (Grant 130497/2009-6). C. M. Denardini thanks CNPq/MCT for the Grants 305242/2011-3 and 470553/2009-0 that partially supported the present study. E. C. thanks the partially support received from $\mathrm{CNPq} / \mathrm{PROANTAR}$ under project no. 0520186/06-0, and from INCT\&APA (CNPq no.: 574018/20085). We thank the students at the Laboratório de Ciências Espaciais de Santa Maria for maintaining the continuous IRIS observations. The authors wish to acknowledge the referees for their assistance in evaluating this paper.

Editor-in-Chief M. Pinnock and Topical Editor P.-L. Blelly thank two anonymous referees for their help in evaluating this paper. 


\section{References}

Abdu, M. A., Degaonkar, S. S., and Ramanathan, K. R.: Attenuation of galactic radio noise at $25 \mathrm{Mhz}$ and 21.3 Mhz in the ionosphere over Ahmedabad during 1957-1964, J. Geophys. Res., 72, 15471554, 1967.

Abdu, M. A., Ananthakrishnan, S., Coutinho, E. F., Krishnan, B. A., and Reis, E. M. da S.: Azimuthal drift and precipitation of electrons into the South Atlantic Geomagnetic Anomaly during an SC Magnetic storm, J. Geophys. Res., 78, 5830-5836, 1973.

Abdu, M. A., Batista, I. S., Carrasco, A. J., and Brum, C. G. M.: South Atlantic Magnetic anomaly ionization: a review and a new focus on electrodynamics effects in the equatorial ionosphere, J. Atmos. Sol.-Terr. Phys., 67, 1643-1657, 2005

Armstrong, R. J., Berkey, F. T., and Melbye, T.: The day to night absorption ratio in auroral zone riometer measurements, Planet. Space Sci., 25, 1193-1198, 1977.

Balsley, B. B. and Ecklund W. L.: A portable coaxiall collinear antenna, IEEE Trans. Antennas Propag., A-P20, 513-516, 1972.

Browne, S., Hargreaves, J. K., and Honary, B.: An imaging riometer for ionospheric studies, Electronics \& Communication Engineering Journal, 7, 209-217, 1995.

Detrick, D. L. and Rosenberg, T. J.: A phased-array radiowave imager for studies of cosmic noise absorption, Radio Sci., 25, 325338,1990 .

Duffett-Smith, P. E.: Practical astronomy, Cambridge University Press, Cambridge, 1979.

Fredriksen, A. and Dyce, R. B.: Ionospheric absorption investigations at Hawaii and Johnston Island, J. Geophys. Res., 65, 1177$1181,1960$.

Heisler, R. and Hower, G. L.: Riometer quiet day curves, J. Geophys. Res., 72, 5485-5490, 1967.

Krishnaswamy, S., Detrick, D. L., and Rosenberg, T.: The inflection point method of determining riometer quiet day curves, Radio Sci., 20, 123-136, 1985.
Little, C. G. and Leinbach, H.: The riometer-a device for continuous measurements of ionospheric absorption, Proceedings of the IRE, 46, 320-325, 1959.

Lusignan, B.: Cosmic noise absorption measurements at Stanford, California, and Pullman, Washington, J. Geophys. Res., 65, 3895-3902, 1960.

Mitra, A. P. and Shain, C. A.: The measurement of ionospheric absorption using observation of $18.3 \mathrm{mc} / \mathrm{s}$ cosmic radio noise, J. Atmos. Terr. Phys., 4, 204-218, 1953.

Nishino, M., Tanaka, Y., Oguti, T., Yamagishi, H., and Holtet, J. A.: Initial observations results with imager riometer at NY-Alesud ( $L=16$ ), NIPR Symp. Upper Atmosphere Physics, 6, 47-61, 1993.

Nishino, M., Makita, K., Yumoto, K., Rodrigues, F. S., Schuch, N. J., and Abdu, M. A.: Unusual ionospheric absorption characterizing energetic electron precipitation into the South Atlantic Magnetic Anomaly, Earth Planets Space, 54, 907-916, 2002.

Nishino, M., Makita, K., Yumoto, K., Miyoshi, Y., Schuch, N. J., and Abdu, M. A.: Energetic particle precipitation in the brazilian geomagnetic anomaly during the "Bastille Day Storm" of July 2000, Earth Planets Space, 58, 607-616, 2006.

Rostoker, G.: Geomagnetic indices, Rev. Geophys. Space Phys., 4 , 935-950, 1972.

Tanaka, Y., Makita, K., Nishino, M., and Ookawa, T.: Development of data analysis program for imaging riometer by using MATLAB, Bulletin of science and engineering, Takushoku University, 10, 61-66, 2007.

Steiger, W. R. and Warmick, J. W.: Observations of cosmic radio noise at 18 mc/s in Hawaii, J. Geophys. Res., 66, 57-66, 1961.

Wrenn, G. L., Rodger, A. S., and Rishbeth, H.: Geomagnetic storms in the Antarctic F-Region. I. Diurnal and seasonal patterns for main phase effects, J. Atmos. Terr. Phys., 49, 901-913, 1987. 